\title{
Functional lesional neurosurgery for tremor - a systematic review and meta-analysis
}

Sebastian R. Schreglmann, MD ${ }^{1,2}$; Joachim K. Krauss, MD ${ }^{3}$; Jin Woo Chang, MD, PhD 4; Kailash P. Bhatia, MD, $\mathrm{FRCP}^{2}$; Georg Kägi, MD ${ }^{1}$

${ }^{1}$ Department of Neurology, Kantonsspital St.Gallen, Rorschacher Strasse 95, 9007 St.Gallen, Switzerland

${ }^{2}$ Sobell Department of Motor Neuroscience and Movement Disorders, Institute of Neurology, UCL, 33 Queen Square, London WC1N 3BG, UK

3 Department of Neurosurgery, Medizinische Hochschule Hannover, Carl-Neuberg-Strasse 1, 30625 Hannover, Germany

${ }^{4}$ Department of Neurosurgery, Yonsei University College of Medicine, 205 Seongsanno, Seodaemun-gu Seoul 120752, Republic of Korea

Corresponding Author:

Georg Kägi, MD

Department of Neurology, Kantonsspital St.Gallen,

Rorschacherstrasse 97, 9000 St.Gallen, Switzerland

Tel: +41 714941111

Fax: +41 714941111

e-mail: georg.kaegi@kssg.ch

$\begin{array}{ll}\text { word count abstract: } & 248 \text { / 250 } \\ \text { word count text: } & 5050 \text { / } 5000 \\ \text { tables/figures: } & 2 / 3 \\ \text { references: } & 92 \\ \text { Funding sources: } & \text { none }\end{array}$




\section{ABSTRACT}

Background: This work evaluates the consistency, effect size and incidence of persistent side effects of lesional neurosurgical interventions in the treatment of tremor due to Parkinson`s disease (PD), Essential Tremor (ET), Multiple Sclerosis (MS) and midbrain lesions.

Methods: Systematic review and meta-analysis according to PRISMA-P guidelines. Random effects meta-analysis of standardized mean difference based on a peer-reviewed protocol (PROSPERO no. CRD42016048049).

Results: From 1249 abstracts screened, 86 peer-reviewed studies reporting 102 cohorts homogeneous for tremor aetiology, surgical target and technique were included.

Effect on PD tremor was better when targeted at the ventral intermediate nucleus (V.im.) by radiofrequency ablation (RF) (Hedge`s g: -4.15;) over V.im. by Gamma knife (GK) (-2.2), subthalamic nucleus (STN) by RF (-1.12) and globus pallidus internus (GPi) by RF (-0.89). For ET MRI-guided focused ultrasound (MRIgFUS) ablation of the cerebellothalamic tract (CTT) (-2.35) and V.im. (-2.08) showed similar mean tremor reductions to V.im. ablation by RF (-2.42) or GK (-2.13). In MS V.im. ablation by GK (-1.96) and RF (-1.63) were similarly effective.

Mean rates of persistent side effects after unilateral lesions in PD were 12.8\% (RF V.im.), 13.6\% (RF STN), 9.2\% (RF GPi), 0.7\% (GK V.im.) and 7.0\% (MRIgFUS V.im.). For ET, rates were 9.3\% (RF V.im.), 1.8\% (GK V.im.), 18.7\% (MRIgFUS V.im.) and 0.0\% (MRIgFUS CTT), for MS 37.7\% (RF V.im.) and for rubral tremor 30.3\% (RF V.im.).

Conclusion: This meta-analysis quantifies safety, consistency and efficacy of lesional neurosurgical interventions for tremor by target, technique and aetiology.

Key words: tremor, thalamotomy, subthalamotomy, pallidotomy

\section{INTRODUCTION}


Tremor due to Essential Tremor (ET), Parkinson's diseae (PD), dystonic tremor, multiple sclerosis (MS) and lesions to the midbrain or cerebellar structures (rubral tremor) frequently shows limited response to oral medication [1-3]. According to current pathophysiological understanding tremor is most likely the result of abnormal central oscillatory activity within a network involving motor cortex, thalamus, globus pallidus and cerebellum[4-6]. Since the 1940ies, therapeutic lesional surgical interventions have aimed at various anatomical structures within this network with variable success[7-10]. The historical development of lesional neurosurgery over the past 8 decades was markedly influenced by the technology available for target location, identification, and lesioning[11]. Accordingly, the advent of in principle reversible deep brain stimulation (DBS) technology[12] by and large replaced irreversible lesional approaches in academic centres for the past 25 years. Although only a single randomized controlled trial on a heterogeneous patient population compared lesional and stimulation approaches head-to-head[13], efficacy was deemed similar between both techniques, while the side effect risk was found to be higher with thalamotomy than DBS[14,15].

Nevertheless, lesional interventions were performed world-wide[16] and appreciated in terms of efficacy[17]. Since the introduction of Gamma Knife (GK)[18], incisionless functional neurosurgery, i.e. lesion placement through the intact skull, became a possibility[9]. Recently, the addition of MRI-guided high-intensity focused ultrasound (MRIgFUS)[19] has again stimulated interest in this field [20,21], as it offers an incisionless approach to create an intracerebral lesion under direct imaging-control in a step-wise manner[22-25]. First data comparing surgical interventions by DBS, GK and MRIgFUS indicate better functional disability improvement with MRIgFUS over DBS and GK, while incisionless approaches show better cost-effectiveness [26]. One could therefore say that these technological advances lead to a renaissance of lesional functional neurosurgery.

A wealth of studies on lesional tremor treatment has been published over time but has never been formally compiled, summarized and assessed in order to estimate consistency of treatment effects or persisting side effects after lesional interventions. In order to guide the renewed interest in lesional neurosurgical interventions for tremor, we summarize the efficacy and safety of lesional interventions by a systematic review and meta-analysis of the existing literature. In particular we aimed to answer the following questions:

- What is the evidence for efficacy of lesional neurosurgical interventions on tremor severity in tremor due to PD, ET, MS and midbrain / rubral origin by different lesioning techniques and targets?

- What is the prevalence rate of persistent side effects after unilateral lesional interventions for different lesioning techniques and targets? 
Different thalamic nomenclature systems have been used and cited for the same structures[27]. Throughout the following we use Hassler's ventral intermediate nucleus (V.im.) analogous to Walker's VIM and the ventral lateral posterior nucleus (VLp) by Jones. Hassler's ventralis oralis anterior nucleus (V.oa.) corresponds to Jones' ventral lateral anterior nucleus (VLa), while the ventralis oralis posterior nucleus (V.op.) is considered a transition zone between VLa and VLp.

Subthalamic structures, containing the radiatio prelemniscalis (Ra.prl), including the cerebellothalamic tract (CTT) and the nucleus of the caudal zona incerta (cZI) as well as the pallidothalamic tract containing ansa lenticularis and the fasciculus lenticularis, are referred to as the posterior subthalamic area (PSA)[11].

\section{METHODS}

This work was conducted according to the recommendations from the Preferred Reporting Items for Systematic Review and Meta-Analysis Protocols (PRISMA-P) 2015 statement[28]. The review protocol has been registered in the International Prospective Register of Systematic reviews (PROSPERO no. CRD4201604804), peer-reviewed and published[29].

\section{Study design and selection criteria}

This study assessed the intervention effect by comparing pre- and post-interventional tremor severity. Cohorts reporting a minimum of five patients of or above the age of 18 with a tremor diagnosis of confirmed aetiology, subjected to unior bilateral lesional functional neurosurgery in a central neuroanatomical structure (thalamus, pallidum, subthalamic nucleus, alternative subcortical targets) by means of an intracerebral lesion, either by incisional (placement of a stylette, leukotome, cryosurgery or radiofrequency (RF) probe after skull opening) or incisionless (MRIgFUS, GK) means. Cases reporting results from mixed aetiologies (subjects of different aetiologies grouped together) or mixed interventions (different techniques, different anatomical targets or more than one target grouped together), as well as non-lesional approaches including DBS, were excluded (see Table 1). 
Table 1. In- and Exclusion criteria

\section{Inclusion criteria}

Population

Study design

Efficacy Outcome

Safety Outcome

Type of publication

Time-frame structure
- Adult patients (>18yrs.) with a tremor diagnosis of confirmed aetiology

- uni- or bilateral lesional functional neurosurgical intervention in a central neuroanatomical

- by incisional (placement of a stylette, leukotome, cryosurgery or radiofrequency probe after skull opening) or incisionless (transcranially focused ultrasound (MRI-guided focused ultrasound (MRIgFUS)), radiation energy (Gamma Knife (GK)) means

- randomized controlled trials (RCTs), Meta-Analysis, case-control, prospective and retrospective case series

- a minimum of five subjects included per cohort (indication / treatment)

- minimum follow-up of 2 months after the intervention

- reporting tremor outcome on a validated tremor scale

- side effects after unilateral only interventions

- cohorts including bilateral interventions

- Letters, abstracts and editorials

Before January 1990

Exclusion criteria

- cases subjected to lesional functional structure at the same time

or stimulation techniques (deep brain stimulation)

- studies reporting results from mixed aetiologies or mixed intervention (different anatomical targets or techniques)

January 1990 - February 2017 
Data from peer-reviewed randomized controlled trials (RCTs), Meta-Analyses, case-control, prospective and retrospective case series were included in the analysis, while letters, abstracts and editorials were not. Due to limitations of earlier reports pertaining to established diagnostic criteria, use of validated clinical assessment tools and target verification[7], we restricted the inclusion to publications/studies from January 1990 onwards. As sometimes more than one group of patients homogeneous for aetiology, technique and target is reported per publication/study, we refer to these homogeneous groups as cohorts throughout this work.

For the efficacy analysis, cohorts reporting upper limb tremor outcome contralateral to the intervention using validated tremor scales were included. PD cohorts reporting tremor in the ON state only as well as cohorts excluding tremordominant PD cases were not included in the analysis (see Figure 1).

For the safety assessment, all cohorts reporting persistent side effects after unilateral only interventions were included mean and standard deviation (SD) of the incidence of all persistent side effects was calculated per group.

\section{Search strategy}

Searches of Medline and the Cochrane databases were conducted without restrictions of publication language. The search terms "tremor*" AND "lesion*”, "neurosurg*”, "thalamotomy", "subthalamotomy", "pallidotomy" were used additional publications were identified from the reference lists of selected papers. Identified abstracts were screened and selected based on reporting human clinical outcome data by one investigator (SRS) - screening for in depth review was performed independently by two investigators (SRS \& GK) and discrepancies were resolved before further analysis. Data was then extracted by using a standardized data collection tool.

\section{Statistical Analysis}

Primary outcome measure was the change in upper limb tremor severity from baseline to follow-up. To minimize selection/reporting bias if more than one follow-up time-point was reported, the time-point with the largest number of patients retained was included in the analysis. The standardized mean difference (Hedges`g) was used to calculate the effect size of the treatment effect, as this transforms original data to a uniform scale and allows to combine data measured with different scales or scale-items[30] to account for reporting heterogeneity in the primary data. Pooled participant data from studies reporting pre- and post-interventional tremor scores (mean \pm SD) were included in a random-effects meta-analysis using the Meta-Essentials workbook4 toolbox[31] and displayed in Forest plots. 
Heterogeneity of subgroups was calculated using the $\mathrm{I}^{2}$ statistic with an $\mathrm{I}^{2}>50 \%$ assumed to represent substantial heterogeneity. Follow-up duration in months was imputed as a moderator and computed by univariate, weighted regression using a random effects model[31] to control for an effect of follow-up duration on the effect size.

Homogeneous cohorts (same tremor aetiology, intervention target and technique) were grouped together for subgroupanalysis if they consisted of a minimum of $n=2$. A formal assessment of publication bias is useful in the presence of a sufficient number $(>10)$ of homogeneous data sets but can be misleading in small and heterogeneous ones[32]. Our preset cut-off for formal bias assessment was if subgroups with more than 10 studies included were shown to have no substantial level of heterogeneity $\left(\mathrm{I}^{2}<50 \%\right)$. A narrative evaluation is provided for all other cases. After testing for normality, safety data was analysed using non-parametric Kruskal-Wallis test and Dunn`s multiple comparison test where appropriate using GraphPad PRISM 6 (La Jolla, CA/USA).

\section{Quality assessment}

Studies included in this meta-analysis were assessed according to standardized tools established for the grading of cohort studies (Newcastle Ottawa-Scale [33]), and randomized controlled trials (Jadad-Scale [34]).

\section{RESULTS}

The database searches (final on January $14^{\text {th }}$ 2017) identified 1249 abstracts, from which 127 studies were selected for screening (see Figure 1). After exclusion of studies not fulfilling in-/exclusion criteria (see Table 1), 86 studies reporting 102 cohorts were reviewed in depth (extracted data are summarized in Supplementary Table 1). According to our standardized quality assessment, the scientific quality of studies included was variable - detailed results are given in supplementary tables 1 and 2 .

The efficacy meta-analysis (Figure 2) is based on data from 1255 patients (46 cohorts), the majority of which suffered from PD (64.2\%) and ET (32.8\%), with MS (2.5\%) and rubral tremor cases (0.4\%) being much less frequently reported. The rate of reported persistent side effects after unilateral lesional interventions (Figure 3) is based on data from 1415 subjects reported in 59 cohorts. Again, patients with PD (71.2\%) were more frequent than with ET (19.3\%), MS (6.4\%) and rubral tremor $(3.0 \%)$.

We have identified studies reporting outcome after interventions in the following categories - subgroup analysis was done if $\geq 2$ studies reported outcome of the same aetiology and target: 1) PD tremor: RF ablation ventral intermedial (V.im.) nucleus, RF ablation Globus pallidus internus (GPi), RF ablation subthalamic nucleus (STN), Leukotome 
ablation GPi, GK ablation V.im.; 2) ET tremor: RF ablation V.im., GK ablation V.im., MRIgFUS ablation V.im., MRIgFUS ablation CTT; 3) MS tremor: RF ablation V.im., GK ablation V.im.; 4) Rubral tremor: RF ablation V.im.. The selected publications used the following standardized and validated clinical scales for tremor quantification: United Parkinson's disease rating scale, part III (UPDRS III)[35], clinical rating scale for tremor (CRST[36,37]), Washington Hights-Inwood genetic study of essential tremor (Whiget) scale[38] and Bain Findley tremor scale[39].

\section{Parkinson's disease tremor}

We identified 17 retrospective, 10 prospective (1 controlled[40]) and one RCT[41] cohorts that fulfilled criteria for efficacy analysis - overall $31.4 \%$ of cases were assessed prospectively. The majority of PD cases received treatment using RF ablation ( $n=677)$, followed by GK ( $n=129)$ (Figure 2A). Anatomically, the majority were treated at the GPi $(n=367)$, with V.im. $(n=304)$ and STN ( $n=135)$ following. Effect on tremor was strongest in the RF V.im. group (Hedge`s g: $-4.15,95 \%$ CI: -5.13 to -3.17$)$ with substantial heterogeneity $\left(\mathrm{I}^{2}=0.77\right)$, followed by GK V.im. $(-2.20,-3.62$ to $\left.-0.78, \mathrm{I}^{2}=0.94\right)$, RF STN $\left(-1.12,-1.4\right.$ to $\left.-0.84 ; \mathrm{I}^{2}=0.38\right)$ and RF GPi $\left(-0.89,-1.16\right.$ to $\left.-0.61, \mathrm{I}^{2}=0.68\right)$. Across cohorts, duration of follow-up did not have a significant influence on effect size (slope $-0.01, \mathrm{p}=0.61$ ).

For the safety analysis 35 cohorts fulfilled inclusion criteria, with the majority of patients being subjected to RF pallidotomy (Figure 3A). The rates of reported persistent side effects after unilateral lesions were $12.8 \pm 19.0 \%$ for RF V.im. ( $n=198), 13.6 \pm 8.2 \%$ for RF STN ( $=150), 9.2 \pm 15.9 \%$ for RF pallidum $(n=514), 0.7 \pm 1.2 \%$ for GK V.im. $(n=126)$ and $7.0 \pm 9.9 \%$ for MRIgFUS V.im. $(n=20)$ lesions - this side effect rate $\left(\chi^{2}=6.022, p=0.20\right)$ as well as the duration of follow-up $\left(\chi^{2}=1.005, \mathrm{p}=0.91\right)$ was not significantly different between groups. One RCT reporting leucotomy lesions in the GPi for PD (n=9) reported no persistent side effects[42].

\section{Essential Tremor}

For the efficacy analysis 6 retrospective and 7 prospective cohorts fulfilled inclusion criteria - overall $64.3 \%$ of cases were assessed prospectively. Lesions were placed at the V.im. using GK $(n=254)$, MRIgFUS $(n=79)$ and RF ( $n=25)$, as well as the CTT using MRIgFUS ( $n=27$ ) (Figure 2B). Mean effect on tremor was similar between groups: MRIgFUS ablation of the cerebellothalamic tract (CTT) $(-2.35 ;-2.51 /-2.19)$ and V.im. (-2.08; -2.77/-1.39) showed similar mean tremor reductions to V.im. ablation by RF $(-2.42 ;-5.26 / 0.43)$ or GK $(-2.13 ;-3.78 /-0.48)$. Across cohorts, duration of follow-up did not have a significant influence on treatment effect size (slope $-0.02, \mathrm{p}=0.28$ ).

For the safety analysis 13 cohorts $(n=273)$ were identified (Figure 3B). The rate of reported persistent side effects after 
unilateral lesions were $9.3 \pm 8.6 \%$ for RF V.im. $(n=32), 1.8 \pm 3.0 \%$ for GK V.im. $(n=153), 18.7 \pm 16.2 \%$ for MRIgFUS V.im. $(n=82)$ and $0.0 \pm 0.0 \%$ for MRIgFUS CTT $(n=6)$ - this was not significantly different between groups $\left(\chi^{2}=4.49\right.$, $\mathrm{p}=0.21$ ). Follow-up duration between studies differed on a group level $\left(\chi^{2}=7.46, \mathrm{p}=0.02\right)$, but not on multiple comparisons (RF V.im. vs. GK V.im. p=0.07, RF V.im. vs. MRIgFUS V.im. p=0.51, RF V.im. vs. MRIgFUS CTT p>0.99, GK V.im. vs. MRIgFUS V.im. p>0.99, GK V.im. vs. MRIgFUS CTT p=0.69, MRIgFUS V.im. vs. MRIgFUS CTT p>0.99).

\section{MS tremor}

Four retrospective cohorts reporting 32 subjects were included in the efficacy meta-analysis. Tremor reduction after RF V.im. $\left(-1.63,-2.56\right.$ to $\left.-0.70, \mathrm{I}^{2}=0.14\right)$ and GK V.im. ablation $\left(-1.96,-3.12\right.$ to $\left.-0.81, \mathrm{I}^{2}=0.32\right)$ were similar (Figure $2 \mathrm{C}$ ). Across cohorts, duration of follow-up did not have a significant influence on effect size (slope $-0.13, \mathrm{p}=0.59$ ).

For the safety analysis six retrospective cohorts of RF V.im. ablation were included (Figure 3C) reporting a persistent side effect rate after unilateral lesions of $37.7 \pm 23.9 \%(n=85)$ - the only GK V.im. study reported no persistent side effects $(n=6)[43]$.

\section{Rubral tremor}

The effect size in one retrospective RF V.im. cohort for rubral tremor fulfilling inclusion criteria was -4.03 (95\% CI 7.68 to $-0.38 ; n=5)$. For the safety analysis four cohorts were identified (Fig. 3C), reporting a persistent side effect rate of $30.3 \pm 10.9 \%(n=43)$.

\section{Dystonic Tremor}

There was no publication on lesional neurosurgery for dystonic tremor found that fulfilled inclusion criteria for this analysis - the therapeutic evidence for this entity, mainly based on small trials and case series, has been reviewed elsewhere[44].

\section{DISCUSSION}

This systematic review summarizes and assesses the efficacy of lesional functional neurosurgical interventions for tremor published since 1990 in a systematic manner. Applying a stringent set of inclusion and exclusion criteria it 
summarizes the available evidence to support the efficacy for each different intervention target and technique. As such it presents the current state of the field and allows comparison between different intervention techniques and anatomical targets according to tremor aetiology.

\section{Efficacy of lesioning for tremor}

Across all tremor aetiologies, the large majority of patients have been treated at the V.im. target and with all but one of the GK studies[45] showing significant improvement, our data confirm the efficacy of this target. In this regard, the PD subgroup analysis is of particular interest, as it confirms the previous perception from decades of functional neurosurgery on the superiority of the V.im. target for tremor suppression. We acknowledge that our analysis does not take other major motor symptoms of PD such as bradykinesia, rigidity and dyskinesia, nor non-motor symptoms into account. As it was, however, designed to quantify treatment efficacy for tremor across aetiologies this is deemed acceptable. We would however expect a selection bias against tremor-dominant cases in the RF GPi group beyond the studies that explicitly mentioned their exclusion[46] or preference of akinetic-rigid cases[47-49], as pallidotomies are historically considered to be less effective for tremor reduction than thalamotomies. Nevertheless, pallidotomies form the largest group $(n=514)$ of PD patients with the highest rate of prospectively sampled data in this analysis. By and large they show a stronger effect size in larger[50-52] than smaller samples[53,54], confirming that pallidotomies have a significant effect on tremor. The significant superiority of RF V.im. over both RF GPi and RF STN ablation for PD tremor reduction (95\% CI intervals non-overlapping) nevertheless confirms the superiority of the thalamic target for tremor reduction in PD and corroborates the importance of this structure in the involved tremor-network.

\section{Thalamic V.im. target}

\section{Essential Tremor}

We were surprised to find only very few data on RF V.im. lesioning in ET published during the time period studied, resulting in the large $95 \%$ confidence interval crossing the midline (Figure $2 \mathrm{~A}$ ). However, given only two studies were included in this subgroup, each showing a significant effect individually, we consider this observation a statistical artefact common to subgroup analyses with a low n. The GK thalamotomy group showed the highest degree of heterogeneity and included both studies reporting the highest[55] and lowest effect size[45] in this work. In part this might reflect the variability of radiation effect on tissue, which still cannot be predicted for individual cases[56]. Given 
that much larger pro-[57] and retrospective[58] studies reported a smaller effect size, the efficacy reported in Kondziolka et al.[55] seems very optimistic. Interestingly, heterogeneity of results was lowest amongst MRIgFUS studies, in particular targeting the CTT $\left(\mathrm{I}^{2}=0.00\right)$, which is surprising given the small number of cases and cohorts. Although a direct comparison between MRIgFUS V.im. vs. CTT is inappropriate given the current number of available cases, it nevertheless indicates at least similar efficacy. Overall the combined effect size for V.im. lesions in ET indicates a robust effect on tremor reduction and corroborates decades of thalamotomy experience. Long-term followup studies report a robust reduction of tremor severity after a mean 59 months using RF[59] and a mean 36-40 months[55,57] using GK V.im. ablation. The longest follow-up of a lesional tremor study has been reported for targeting of the ZI + V.oa./V.op./Ra.prl at 8.5 years of follow-up[60], although this study of combined targets is not included in the efficacy meta-analysis.

Our analysis revealed different rates of persistent side effects of unilateral lesional treatment at the V.im. target according to surgical technique: while overall the rate recorded was highest for MRIgFUS V.im. lesions (18.7\%), severe effects such as hemiparesis were reported in 9.3\% after RF V.im., $1.8 \%$ after GK V.im. and 1.2\% after MRIgFUS V.im. ablation, while paraesthesia accounted for $60 \%$ of persistent side effects after MRIgFUS V.im. treatment. Importantly, the rate of prospectively monitored patients was much higher in MRIgFUS V.im. (100\%) than in RF V.im. (28\%) or GK V.im. (12\%) studies, possibly introducing recall bias in the latter. Taking these mean values as a benchmark illustrates how the dysarthria rate after unilateral V.im. lesions (RF: $0 \%, n=32$; GK: 1.3\%, $n=153$; Figure 3B) drastically increases with simultaneous, bilateral treatment (RF: 66\%, n=3 [61]).

\section{Parkinson's disease tremor}

It is difficult to explain the much larger effects size of RF V.im. (Hedge`s g -4.15) over GK V.im. (-2.20) lesioning in PD, especially as the effect size of V.im. lesions in ET (-2.34) is similar to the latter. Unfortunately, primary data do not allow to compare baseline tremor severities between studies and groups, making it difficult to ascertain the reason for this difference. It is a possibility that electrophysiological target verification, used in all of RF V.im. PD cases but in only a fraction of RF V.im. ET and none of the GK V.im. PD cases included, may have contributed to this difference. Still, the relative consistent outcome in the well sized RF V.im. group supports the validity of this finding.

Unilateral lesioning using RF ablation appeared to cause similar rates of persistent side effects between 9.2-13.6\% if aiming at the V.im. (mainly hemiparesis, dysarthria, gait disturbance), STN (mainly hemiballism, dyskinesia) and the pallidum (mainly hemiparesis, dysarthria, anopsia), while incisionless interventions and in particular GK caused 
somewhat less frequent side effects. Comparing the mean incidence rate of persistent dysarthria \& dysphagia after pallidal unilateral $(2.5 \%, \mathrm{n}=515$, Figure 3A) and bilateral $(11 \%, \mathrm{n}=28$ [62]; 43\%, $\mathrm{n}=7$ [63]), as well as RF V.im. thalamic unilateral $(1.5 \%, \mathrm{n}=198$, Figure $3 \mathrm{~A})$ and bilateral $(11 \%, \mathrm{n}=9[64] ; 50 \%, \mathrm{n}=2[59])$ quantifies the dramatically increased risk with bilateral treatment.

\section{MS tremor}

MS tremor differs from the above-mentioned aetiologies as its phenotype depends on the lesion location. Our data indicate a robust positive effect of V.im. lesional surgery (Figure 2C). However, efficacy seems much more short-lived than in other aetiologies and the mean tremor-free interval after V.im. ablation or stimulation has been reported to be as short as 3 months only[65]. Two retrospective, controlled studies suggest that thalamotomy might be superior to thalamic stimulation on longer follow-up [66,67].

Furthermore, our analysis suggests persistent side effects of unilateral lesional interventions in up to $40 \%$ of cases. In interpreting both the modest results and higher rate of side effects, the advanced disease stages of MS patients subjected to surgery have to be taken into account[43,68,69]. Caution should therefore be exercised in patients presenting with tremor and ataxia, as thalamotomy might improve tremor but aggravate ataxia, compromising limb dysfunction[13,70] and even worsening quality of life[70] or manual skills[43]. The limited number of reported cases of MS relapses after GK treatment do not allow to conclude that irradiation has a causal relationship with demyelinating events, although MS patients have a higher susceptibility for demyelinating events after whole brain irradiation[43]. Overall, lesional therapy for tremor appears to be a therapeutic option in only a limited, highly selected subset of MS tremor patients, particularly with low disease activity and little ataxia.

\section{Rubral tremor}

Injury to the cerebellum, midbrain or thalamus containing the afferent cerebellar fibres to the thalamus are causal in the generation of midbrain/rubral tremor[71]. Our analysis confirms the paucity of data on lesional treatment for this entity, for which historically the majority of patients have been treated at the V.im. target[72]. In the past, authors repeatedly pointed out that spontaneous improvement of tremor may occur within 12 months of the initial injury, which therefore should have lapsed before planning an intervention[73,74]. This and the $30 \%$ incidence rate of persistent side-effects shown in our analysis preclude lesional treatment to highly selected cases. There is only sparse evidence to support 
lesional treatment of symptomatic tremors due to Wilson`s disease, PKAN and other rare aetiologies[75].

\section{Subthalamic nucleus target}

Among the targets employed for PD tremor, the high level of consistency of tremor improvement after RF STN ablation studies $\left(I^{2}=0.38\right)$ points at a very reliable and predictable, although not very strong effect of this target. Although theoretically elegant, STN lesioning has never been widely practised in the field, mainly due to the risk of causing hemiballism and -dyskinesia. Our side effect analysis $(n=140$, figure $3 \mathrm{~A})$ suggests a mean rate of $9.3 \%$ of persistent hemiballism/-dyskinesia after unilateral lesional STN treatment in PD. This increases with bilateral treatment up to $20 \%$ $(n=5[76])$. While some argue the risk for surgery-induced dyskinesia to be smaller if a STN lesion involves also dorsally located pallidofugal fibres in the ZI[46,77,78], others reported dyskinesia to be associated with larger lesion size[79-81]. In general, the effect on tremor after STN lesions has been described to decrease over time[79-81]. Some authors also suggested that a predominant improvement of tremor might actually be due to lesion encroachment on the adjacent ZI and even V.im.[82], as confirmed on post-mortem analysis[78].

Certainly, the STN proper as a neurosurgical target is limited to PD only and although the effect on bradykinesia and rigidity are appreciated, our analysis confirms its effect on tremor to be less powerful than that of thalamic targets.

\section{Incisionless approaches}

Incisionless approaches using GK or MRIgFUS for functional neurosurgery appeal due to their apparent noninvasiveness. Indeed, they clearly circumvent the risks associated with open brain surgery or implantation of devices but their technique-inherent advantages and disadvantages should be weighed carefully. Furthermore, at least for MRIgFUS, the evidence base for its efficacy and safety is promising but still limited.

In ET our analysis shows that the efficacy of GK and MRIgFUS ablation of thalamic/PSA targets is comparable to established RF V.im. ablation. Interestingly, both the number of cases ( $n=358$ vs. $n=25$ ) as well as the percentage of prospective cases $(74 \%$ vs. $0 \%)$ was much higher among incisionless treated patients, pointing that the latter has possibly by en large replaced RF ablation for ET at least in academic centres. Within the group of incisionless interventions, the effect size was found to be much more heterogeneous with GK V.im. $\left(\mathrm{I}^{2}=0.92\right)$ than MRIgFUS V.im. $\left(I^{2}=0.51\right)$ or MRIgFUS CTT $\left(I^{2}=0.00\right)$ ablation, although the prior is based on a larger number of and higher rate of 
prospectively assessed patients. This indicates a technique-inherent heterogeneity related to GK.

Persistent mild contralateral paresis, hypaesthesia, dysarthria and dysphagia have been reported after GK lesioning, but overall this technique caused the lowest rate of persistent side effects both in PD and ET, although this is also based on cohorts with the lowest rate of prospective follow-up. The unexpected enlargement of lesions documented up to 14 months after treatment even with moderate doses of 140Gy[45] argues for adherence to follow-up durations long enough to cover the evolution of potential side effects - therefore, we suggest that follow-up in GK trials should be at least 14 months. Given the natural evolution of GK lesions with time, 6 months follow-up[83] seems insufficient.

Although so far data of only 126 tremor patients have been published in series of MRIgFUS ablation[23-25,84-87], the majority of them have been treated in prospective trials. Comparison of treatment effect sizes, so far only possible for ET, shows similar efficacy for MRIgFUS and RF ablation. MRIgFUS V.im. lesions however entail a comparably high rate of persistent side effects, although this in the majority of cases refers to paraesthesia only (see Figure 3B). The only randomized sham-controlled, multicentre, blind-assessed trial of MRIgFUS V.im. ablation[84] reported persistent paraesthesia in $14 \%$, gait disturbance in $9 \%$, contralateral paresis in $2 \%$, dysmetria in $4 \%$ and disequilibrium in $2 \%$ of cases 12 months after the intervention. In comparison, the side effect rate of MRIgFUS CTT treatment, even when including three cases of staged bilateral treatment, is lower[25,85] and does not include paraesthesia. This can be seen as early indication that the larger anatomical distance of the CTT to eloquent structures might translate into a lower side effect rate.

So far the limited number of patients and short follow-up published for V.im. MRIgFUS interventions in PD[86,87] await replication. The use of conventional thalamotomy to treat PD in the $21^{\text {st }}$ century is in our opinion however limited to exceptional cases, in which tremor is the predominant symptom.

\section{Posterior subthalamic area target}

Mundinger's subthalamotomy appeals to investigators today again. Initially intended to minimize the lesion size necessary to create sufficient effect and increase the anatomical safety margin to eloquent structures near by, over the years considerable evidence accumulated in the DBS literature to suggest that stimulation within the PSA (cZI and Ra.prl) might be superior over V.im. stimulation in ET[88-92] and PD[93]. In the past, neurosurgeons had adapted the idea of targeting this area, as it contains the CTT, containing the connecting fibre tracts between cerebellum and thalamus. In the PSA these fibres are at their highest anatomical density and at a larger Euclidian distance to vulnerable structures such as sensory thalamic nuclei or the internal capsule, providing a rationale to target them at this location. 
Our analysis provides first evidence that lesioning of the CTT[25,85] compared to V.im.[23,24,84] using the same MRIgFUS technique might be of equal effect size and possibly at a smaller rate of persistent side effects. Of course, this will have to be confirmed by additional, well-designed studies. Nevertheless, the observation of no paraesthesia occurrence after CTT lesioning can be seen as confirmation of the use of a larger Euclidian safety margin in particular to thalamic sensory nuclei. Importantly, this indicates a target- and not technique-specific side effect profile for MRIgFUS interventions. Further exploration of this target seems appropriate and needed.

\section{Methodological considerations}

When interpreting the results of this meta-analysis, several factors call for consideration. Although applying a rigid set of in- and exclusion criteria to ensure data homogeneity, validity and reliability, inconsistencies in the included primary data necessitate discussion.

The heterogeneity of targeting and operating technique, inevitably slightly different from one surgical team to another, introduces variability, for which it is impossible to control for. In particular, this applies to targeting accuracy, which of course is one of the most important parameters in functional neurosurgery, which however in most instances is not verified independently. Furthermore, retrospective studies are known to introduce potential recall bias. As indicated in Figures 2 and 3 the proportion of pro- and retrospectively assessed cases differed considerably between tremor aetiologies, techniques and surgical targets. Although the degree of variability introduced by this is difficult to quantify, results in groups with a higher degree of prospective cases may be viewed as more reliable.

Particular attention should also be paid to the fact that primary tremor data was heterogeneous in its use of rating scales, items and compound item sums. Ideally, all studies included would have used the same scale and item, with comparable baseline tremor severity between all study cohorts. As the primary data available was not structured in this way (see Supplementary Table 1) our analysis after data normalization to Hedges`g cannot differentiate between "resting", "kinetic" or "postural" tremor components or their combination, but reports the change in overall tremor severity. We posit that inherent to aetiology this naturally would pertain more to postural and kinetic aspects in ET and more to rest tremor in PD etc. Furthermore, the analysis does not allow for comparison between baseline tremor severity to match for different disease severity, which is impossible given the primary data available. This method however allows for comparison of the effect size in accordance to empiric findings that tremor scales are not linear but logarithmic measures of tremor severity[94].

As another potential weakness, follow-up time-points were not homogeneous between cohorts. Although therefore the 
mean follow-up between cohorts included in the meta-analysis may differ, our analysis with follow-up as a nuisance factor did not provide evidence that this significantly influenced the effect size in any category studied. This should not be interpreted as evidence that the effect size of the discussed interventions does not change over time, which cannot be conclusively answered from the data available.

Using the heterogeneity index $\mathrm{I}^{2}$ as a measure of consistency, summing up all of the above discussed potential causes of heterogeneity, our meta-analysis results for MRIgFUS CTT for ET $\left(\mathrm{I}^{2}=0.00\right)$, V.im. ablation using $\mathrm{RF}\left(\mathrm{I}^{2}=0.14\right)$ and GK for rubral tremor $\left(\mathrm{I}^{2}=0.32\right)$ and $\mathrm{RF} \mathrm{STN}$ for PD tremor $\left(\mathrm{I}^{2}=0.38\right)$ showed the most consistent results.

In addition to the above, this work highlights systematic shortcomings in the functional neurosurgery literature. For example it is impossible to calculate the rate of tremor recurrence, as this is mentioned and quantified in only a fraction of publications. The scarcity of RCTs in our primary data is another important point. Furthermore, the quantification of quality of life and functional impairment, established in many other fields of movement disorder research, is barely reported on in the functional neurosurgical literature. Only prospective, blind-assessed, controlled trials with adequately designed follow-up will answer these important questions.

\section{CONCLUSION}

Results of this meta-analysis confirm the prime role of thalamotomies in lesional surgery for various tremor aetiologies. Theoretical advantages and promising clinical data with regards to safety and efficacy of incisionless procedures currently build up momentum to reappraise lesional interventions as treatment strategies for tremor disorders. This analysis provides evidence in ET to suggest that in particular the rate of persistent side effects is target- and not technique-specific. The exciting technical possibilities of GK and especially MRIgFUS therefore should be geared not only to close the gaps in the existing literature highlighted but also to further advance our knowledge base for treatment decisions. Nevertheless, this systematic review also shows how limited the evidence base is in particular for MRIgFUS ablation so far. It therefore highlights the need for adequately designed prospective trials to support the existing data on safety and efficacy not only for established targets such as V.im., but also of recently rediscovered targets within the PSA. Before that, the indiscriminate application of incisionless interventions to novel indications could potentially harm the further development of this fascinating technique. 
This work is not able to answer the ultimate question in how far the treatment of tremor in the future should rely on stimulation or lesional techniques but it aims to provide the basis for future studies to answer this question. Most likely, tremor aetiology and the target used will influence this decision in addition to available resources and patient preference. Ultimately, however, only head-to-head comparison of DBS and MRIgFUS will be able to answer this question. Similarly, it remains to be seen in how far incisionless technology might be able to reduce the so far inacceptable high side effect rates of bilateral lesional treatment.

\section{Figure legends:}

Figure 1: PRISMA Flow chart of study selection.

Figure 2: Forest plot of data on treatment for Parkinson`s disease tremor (A), Essential Tremor (B) and Multiple Sclerosis tremor (C) included in the meta-analysis. The effect size (Hedge`s g) including 95\% CI is shown for each individual study, as well as for subgroups of homogeneous indication and intervention. Combined effect sizes were calculated for Essential Tremor and MS tremor, as all lesions aimed at the V.im., whereas in Parkinson`s disease this was omitted due to target heterogeneity. The solid vertical line at zero indicates no effect, a negative effect size indicates improvement of tremor, whereas a positive effect size indicates tremor worsening. References for the studies are provided in the appendix.

Figure 3: Rate of reported persistent side effects after unilateral lesional functional neurosurgical interventions for Parkinson`s disease tremor (A), Essential Tremor (B) and MS/Rubral tremor (C). Mean percentages ( \pm standard deviation) of persistent side effects were calculated per target/technique and compared using non-parametric statistics. Additional information on the percentage of subjects assessed prospectively as well as the nature of side effects per group are given in the table below (* indicates statistically different at group level but not on multiple testing).

Table 1: In- and exclusion criteria.

\section{Supplementary files:}


Suppl_Table 1: Characteristics and details of studies included in the systematic review and meta-analysis, reporting tremor severity using validated (A) or non-validated tremor scales (B). Studies reporting tremor scale data. References for the studies are provided in the appendix. * data included in efficacy meta-analysis; $†$ data included in safety analysis of reported persistent side effect after unilateral lesioning; + number of subjects with complete efficacy data (of number of subjects subjected to intervention); data on bilateral interventions in italics; n.r. not reported; - data from same cohort but different time point; retro - retrospective; pro - prospective; ? - not given;

Suppl_Table 2: Jadad quality assessment of randomized controlled trials included in this meta-analysis.

Suppl_Table 3: Newcastle Ottawa quality assessment of cohort studies included in this meta-analysis.

Acknowledgements: SRS is supported by grants by the Swiss Science Foundation, the Swiss Neurological Society and the European Academy of Neurology. We would like to thank Ashwani Jha for helpful discussions on statistical analysis.

Competing interests: Authors declare no competing interests.

Funding: This study received no dedicated funding.

Contributors: SRS performed the systematic review, the statistical analysis and wrote the manuscript. JKK and JWC critically reviewed the manuscript. KPB provided input on data analysis and manuscript structure and critically reviewed the manuscript. GK initiated and supervised the project, was involved in the systematic review process and critically reviewed the manuscript. All authors agreed on the final manuscript draft.

\section{REFERENCES:}

1 Schneider SA, Deuschl G. The treatment of tremor. Neurotherapeutics 2014;11:128-38. doi:10.1007/s13311-0130230-5

2 Lyons KE, Pahwa R. Pharmacotherapy of essential tremor : an overview of existing and upcoming agents. CNS Drugs 2008;22:1037-45. doi:10.2165/0023210-200822120-00006

3 Deuschl G, Raethjen J, Hellriegel H, et al. Treatment of patients with essential tremor. The Lancet Neurology 2011;10:148-61. doi:10.1016/S1474-4422(10)70322-7

4 Raethjen J, Muthuraman M. Cause or compensation? Complex changes in cerebello-thalamo-cortical networks in 
pathological action tremor. Brain 2015;138:2808-10. doi:10.1093/brain/awv238

5 Helmich RC, Janssen MJR, Oyen WJG, et al. Pallidal dysfunction drives a cerebellothalamic circuit into Parkinson tremor. Ann Neurol 2011;69:269-81. doi:10.1002/ana.22361

6 Cagnan H, Little S, Foltynie T, et al. The nature of tremor circuits in parkinsonian and essential tremor. Brain 2014;137:3223-34. doi:10.1093/brain/awu250

7 Guridi J, Lozano AM. A brief history of pallidotomy. Neurosurgery 1997;41:1169-80-discussion1180-3.

8 Higuchi Y, Matsuda S, Serizawa T. Gamma knife radiosurgery in movement disorders: Indications and limitations. Mov Disord 2016;:n/a-n/a. doi:10.1002/mds.26625

9 Campbell AM, Glover J, Chiang VLS, et al. Gamma knife stereotactic radiosurgical thalamotomy for intractable tremor: A systematic review of the literature. Radiotherapy and Oncology 2015;114:296-301. doi:10.1016/j.radonc.2015.01.013

10 Gildenberg PL, Krauss JK. 4 A-01 History of Stereotactic Surgery. In: Textbook of sterotactic and functional Neurosurgery. J. Springer 2008. 1-32.

11 Schreglmann SR, Krauss JK, Chang JW, et al. Functional lesional neurosurgery for tremor: back to the future? J Neurol Neurosurg Psychiatr Published Online First: 21 December 2017. doi:10.1136/jnnp-2017-316301

12 Benabid AL, Pollak P, Gervason C, et al. Long-term suppression of tremor by chronic stimulation of the ventral intermediate thalamic nucleus. The Lancet 1991;337:403-6. doi:10.1007/s00125-015-3676-7

13 Schuurman PR, Bosch DA, Bossuyt PM, et al. A comparison of continuous thalamic stimulation and thalamotomy for suppression of severe tremor. N Engl J Med 2000;342:461-8.

doi:10.1056/NEJM200002173420703

14 Tasker RR. Deep brain stimulation is preferable to thalamotomy for tremor suppression. Surg Neurol 1998;49:145-53-discussion153-4.

15 Pahwa R, Lyons KE, Wilkinson SB, et al. Comparison of thalamotomy to deep brain stimulation of the thalamus in essential tremor. Mov Disord 2001;16:140-3.

16 Jourdain VA, Schechtmann G. Health economics and surgical treatment for Parkinson's disease in a world perspective: results from an international survey. Stereotact Funct Neurosurg 2014;92:71-9. doi:10.1159/000355215

17 Zesiewicz TA, Elble RJ, Louis ED, et al. Evidence-based guideline update: treatment of essential tremor: report of the Quality Standards subcommittee of the American Academy of Neurology. Neurology 2011;77:1752-5. doi:10.1212/WNL.0b013e318236f0fd

18 Leksell L. Cerebral Radiosurgery I. Gammathalamotomy in two cases of intractable pain. Acta Chir Scand 1968;134:585-95.

19 Martin E, Jeanmonod D, Morel A, et al. High-intensity focused ultrasound for noninvasive functional neurosurgery. Ann Neurol 2009;66:858-61. doi:10.1002/ana.21801

20 Hariz M. Focused ultrasound thalamotomy improves essential tremor. Mov Disord 2013;28:1803. doi:10.1002/mds. 25695

21 Deuschl G. New hope for severe essential tremor? The Lancet Neurology 2013;12:420-2. doi:10.1016/S14744422(13)70062-0

22 Lipsman N, Schwartz ML, Huang Y, et al. MR-guided focused ultrasound thalamotomy for essential tremor: a proof-of-concept study. The Lancet Neurology 2013;12:462-8. doi:10.1016/S1474-4422(13)70048-6

23 Elias JW, Huss D, Voss T, et al. A pilot study of focused ultrasound thalamotomy for essential tremor. $N$ Engl J 
24 Chang WS, Jung HH, Kweon EJ, et al. Unilateral magnetic resonance guided focused ultrasound thalamotomy for essential tremor: practices and clinicoradiological outcomes. J Neurol Neurosurg Psychiatr 2015;86:257-64. doi:10.1136/jnnp-2014-307642

25 Schreglmann SR, Bauer R, Hägele-Link S, et al. Unilateral cerebellothalamic tract ablation in essential tremor by MRI-guided focused ultrasound. Neurology 2017;88:1329-33. doi:10.1212/WNL.0000000000003795

26 Ravikumar VK, Parker JJ, Hornbeck TS, et al. Cost-effectiveness of focused ultrasound, radiosurgery, and DBS for essential tremor. Mov Disord Published Online First: 3 April 2017. doi:10.1002/mds.26997

27 Krack P, Dostrovsky J, Ilinsky I, et al. Surgery of the motor thalamus: problems with the present nomenclatures. Mov Disord 2002;17 Suppl 3:S2-8. doi:10.1002/mds.10136

28 Shamseer L, Moher D, Clarke M, et al. Preferred reporting items for systematic review and meta-analysis protocols (PRISMA-P) 2015: elaboration and explanation. BMJ 2015;349:g7647. doi:10.1136/bmj.g7647

29 Schreglmann SR, Krauss JK, Chang JW, et al. Functional lesional neurosurgery for tremor-a protocol for a systematic review and meta-analysis. BMJ Open 2017;7:e015409. doi:10.1136/bmjopen-2016-015409

30 Higgins J, Green S, editors. The Cochrane Handbook for Systematic Reviews of Interventions. In: Cochrane Handbook for Systematic Reviews of Interventions. 2011. www.handbook.cochrane.org

31 van Rhee HJ, Suurmond R, Hak T. User manual for Meta-Essentials: Workbooks for meta-analysis (Version 1.0). www.erim.eur.nlresearch-supportmeta-essentials. 2015.

32 Lau J, Ioannidis JPA, Terrin N, et al. The case of the misleading funnel plot. BMJ 2006;333:597-600. doi:10.1136/bmj.333.7568.597

33 Wells G, Shea B, OConnell D. The Newcastle-Ottawa Scale (NOS) for assessing the quality if nonrandomized studies in meta-analyses. http://www.ohri.ca/programs/clinical_epidemiology/oxford.asp (accessed 21 Oct2016).

34 Jadad AR, Moore RA, Carroll D. Assessing the quality of reports of randomized clinical trials: is blinding necessary? Control Clin Trials 1996;:1-12.

35 Stern MB. The clinical characteristics of Parkinson's disease and parkinsonian syndromes: diagnosis and assessment. In: Stern MB, Hurtig HI, eds. The Comprehensive Management of Parkinson's Disease. New York, NY: : PMA Publishing Corp 1978. 34-9.

36 Fahn S, Tolosa E, Marin C. Clinical rating scale for tremor. In: Jankovic J, Tolosa E, eds. Parkinsons Diseae and Movement Disorders. Munich: 1988. 225-34.

37 Stacy MA, Elble RJ, Ondo WG, et al. Assessment of interrater and intrarater reliability of the Fahn-Tolosa-Marin Tremor Rating Scale in essential tremor. Mov Disord 2007;22:833-8. doi:10.1002/mds.21412

38 Louis ED, Wendt KJ, Albert SM, et al. Validity of a performance-based test of function in essential tremor. Arch Neurol 1999;56:841-6.

39 Bain PG, Findley LJ, Atchison P, et al. Assessing tremor severity. Journal of Neurology, Neurosurgery \& Psychiatry 1993;56:868-73.

40 Merello M, Nouzeilles MI, Cammarota A, et al. Comparison of 1-Year Follow-up Evaluations of Patients with Indication for Pallidotomy Who Did Not Undergo Surgery versus Patients with Parkinson's Disease Who Did Undergo Pallidotomy: A Case Control Study. Neurosurgery 1999;44:461-7-discussion467-8.

41 Vitek JL, Bakay RAE, Freeman A, et al. Randomized trial of pallidotomy versus medical therapy for Parkinson's disease. Ann Neurol 2003;53:558-69. doi:10.1002/ana.10517 
electrical stimulation of the globus pallidus internus in advanced Parkinson's disease. Stereotact Funct Neurosurg 2006;84:64-71. doi:10.1159/000094034

43 Mathieu D, Kondziolka D, Niranjan A, et al. Gamma knife thalamotomy for multiple sclerosis tremor. Surg Neurol 2007;68:394-9. doi:10.1016/j.surneu.2006.11.049

44 Fasano A, Bove F, Lang AE. The treatment of dystonic tremor: a systematic review. J Neurol Neurosurg Psychiatr 2014;85:759-69. doi:10.1136/jnnp-2013-305532

45 Lim S-Y, Hodaie M, Fallis M, et al. Gamma knife thalamotomy for disabling tremor: a blinded evaluation. Arch Neurol 2010;67:584-8. doi:10.1001/archneurol.2010.69

46 Lozano AM, Lang AE, Galvez-Jimenez N, et al. Effect of GPi pallidotomy on motor function in Parkinson's disease. The Lancet 1995;346:1383-7.

47 Laitinen LV, Bergenheim AT, Hariz MI. Leksell"s posteroventral pallidotomy in the treatment of Parkinson"s disease. Journal of Neurosurgery 1992;76:53-61. doi:10.3171/jns.1992.76.1.0053

48 Dogali M, Fazzini E, Kolodny E, et al. Stereotactic ventral pallidotomy for Parkinson's disease. Neurology 1995;45:753-61.

49 Favre J, Burchiel KJ, Taha JM, et al. Outcome of unilateral and bilateral pallidotomy for Parkinson's disease: patient assessment. Neurosurgery 2000;46:344-53-discussion353-5.

50 Lai EC, Jankovic J, Krauss JK, et al. Long-term efficacy of posteroventral pallidotomy in the treatment of Parkinson's disease. Neurology 2000;55:1218-22.

51 Kondziolka D, Bonaroti E, Baser S, et al. Outcomes after stereotactically guided pallidotomy for advanced Parkinson's disease. Journal of Neurosurgery 1999;90:197-202. doi:10.3171/jns.1999.90.2.0197

52 Lang AE, Lozano AM, Montgomery E, et al. Posteroventral medial pallidotomy in advanced Parkinson's disease. N Engl J Med 1997;337:1036-42. doi:10.1056/NEJM199710093371503

53 Baron MS, Vitek JL, Bakay RA, et al. Treatment of advanced Parkinson's disease by posterior GPi pallidotomy: 1-year results of a pilot study. Ann Neurol 1996;40:355-66. doi:10.1002/ana.410400305

54 Yen C-P, Wu S-J, Su Y-F, et al. Unilateral Stereotactic Posteroventral Globus Pallidus Internus Pallidotomy for Parkinson's Disease: Surgical Techniques and 2-Year Follow-Up. The Kaohsiung Journal of Medical Sciences 2005;21:1-8. doi:10.1016/S1607-551X(09)70269-4

55 Kondziolka D, Ong JG, Lee JYK, et al. Gamma Knife thalamotomy for essential tremor. Journal of Neurosurgery 2008;108:111-7. doi:10.3171/JNS/2008/108/01/0111

56 Witjas T, Carron R, Krack P, et al. A prospective single-blind study of Gamma Knife thalamotomy for tremor. Neurology 2015;85:1562-8. doi:10.1212/WNL.0000000000002087

57 Young RF, Li F, Vermeulen S, et al. Gamma Knife thalamotomy for treatment of essential tremor: long-term results. Journal of Neurosurgery 2010;112:1311-7. doi:10.3171/2009.10.JNS09332

58 Kooshkabadi A, Lunsford LD, Tonetti D, et al. Gamma Knife thalamotomy for tremor in the magnetic resonance imaging era. Journal of Neurosurgery 2013;118:713-8. doi:10.3171/2013.1.JNS121111

59 Jankovic J, Cardoso F, Grossman RG, et al. Outcome after Stereotactic Thalamotomy for Parkinsonian, Essential, and Other Types of Tremor. Neurosurgery 1995;37:680-6-discussion686-7.

60 Mohadjer M, Goerke H, Milios E, et al. Long-term results of stereotaxy in the treatment of essential tremor. Stereotact Funct Neurosurg 1990;54-55:125-9.

61 Zirh A, Reich SG, Dougherty PM, et al. Stereotactic thalamotomy in the treatment of essential tremor of the upper extremity: reassessment including a blinded measure of outcome. Journal of Neurology, Neurosurgery \& 
Psychiatry 1999;66:772-5.

62 Shima F, Ishido K, Sun SJ, et al. Surgical control of akinesia in Parkinson's disease. Eur Neurol 1996;36 Suppl 1:55-61.

63 Giller CA, Dewey RB, Ginsburg MI, et al. Stereotactic pallidotomy and thalamotomy using individual variations of anatomic landmarks for localization. Neurosurgery 1998;42:56-62-discussion62-5.

64 Moriyama E, Beck H, Miyamoto T. Long-term results of ventrolateral thalamotomy for patients with Parkinson's disease. Neurol Med Chir (Tokyo) 1999;39:350-6-discussion356-7.

65 Hassan A, Ahlskog JE, Rodriguez M, et al. Surgical therapy for multiple sclerosis tremor: a 12-year follow-up study. European Journal of Neurology 2012;19:764-8. doi:10.1111/j.1468-1331.2011.03626.x

66 Schuurman PR, Bosch DA, Merkus MP, et al. Long-term follow-up of thalamic stimulation versus thalamotomy for tremor suppression. Mov Disord 2008;23:1146-53. doi:10.1002/mds.22059

67 Bittar RG, Hyam J, Nandi D, et al. Thalamotomy versus thalamic stimulation for multiple sclerosis tremor. J Clin Neurosci 2005;12:638-42. doi:10.1016/j.jocn.2004.09.008

68 Alusi SH, Aziz TZ, Glickman S, et al. Stereotactic lesional surgery for the treatment of tremor in multiple sclerosis: a prospective case-controlled study. Brain 2001;124:1576-89.

69 Shahzadi S, Tasker RR, Lozano A. Thalamotomy for essential and cerebellar tremor. Stereotact Funct Neurosurg 1995;65:11-7.

70 Matsumoto J, Morrow D, Kaufman K, et al. Surgical therapy for tremor in multiple sclerosis: an evaluation of outcome measures. Neurology 2001;57:1876-82.

71 Raina GB, Cersosimo MG, Folgar SS, et al. Holmes tremor: Clinical description, lesion localization, and treatment in a series of 29 cases. Neurology 2016;86:931-8. doi:10.1212/WNL.0000000000002440

72 Goldman MS, Kelly PJ. Symptomatic and functional outcome of stereotactic ventralis lateralis thalamotomy for intention tremor. Journal of Neurosurgery 1992;77:223-9. doi:10.3171/jns.1992.77.2.0223

73 Marks PV. Stereotactic surgery for post-traumatic cerebellar syndrome: an analysis of seven cases. Stereotact Funct Neurosurg 1993;60:157-67.

74 Krauss JK, Mohadjer M, Nobbe F, et al. The treatment of posttraumatic tremor by stereotactic surgery. Symptomatic and functional outcome in a series of 35 patients. Journal of Neurosurgery 1994;80:810-9. doi:10.3171/jns.1994.80.5.0810

75 Dwarakanath S, Zafar A, Yadav R, et al. Does lesioning surgery have a role in the management of multietiological tremor in the era of Deep Brain Stimulation? Clin Neurol Neurosurg 2014;125:131-6. doi:10.1016/j.clineuro.2014.07.016

76 Merello M, Tenca E, Lloret SP, et al. Prospective randomized 1-year follow-up comparison of bilateral subthalamotomy versus bilateral subthalamic stimulation and the combination of both in Parkinson's disease patients: a pilot study. Br J Neurosurg 2009;22:415-22. doi:10.1080/02688690801971667

77 Tseng H-M, Su PC, Liu H-M, et al. Bilateral subthalamotomy for advanced Parkinson disease. Surg Neurol 2007;68 Suppl 1:S43-50-discussionS50-1. doi:10.1016/j.surneu.2007.05.058

78 Patel NK, Heywood P, O'Sullivan K, et al. Unilateral subthalamotomy in the treatment of Parkinson's disease. Brain 2003;126:1136-45. doi:10.1093/brain/awg111

79 Su PC, Tseng H-M, Liu H-M, et al. Subthalamotomy for advanced Parkinson disease. Journal of Neurosurgery 2002;97:598-606. doi:10.3171/jns.2002.97.3.0598

80 Su PC, Tseng H-M, Liu H-M, et al. Treatment of advanced Parkinson's disease by subthalamotomy: one-year 
results. Mov Disord 2003;18:531-8. doi:10.1002/mds.10393

81 Alvarez L, Macias R, Lopez G, et al. Bilateral subthalamotomy in Parkinson's disease: initial and long-term response. Brain 2005;128:570-83. doi:10.1093/brain/awh397

82 Parkin S, Nandi D, Giladi N, et al. Lesioning the Subthalamic Nucleus in the Treatment of Parkinson's Disease. Stereotact Funct Neurosurg 2001;77:68-72. doi:10.1159/000064599

83 Niranjan A, Kondziolka D, Baser S, et al. Functional outcomes after gamma knife thalamotomy for essential tremor and MS-related tremor. Neurology 2000;55:443-6.

84 Elias WJ, Lipsman N, Ondo WG, et al. A Randomized Trial of Focused Ultrasound Thalamotomy for Essential Tremor. N Engl J Med 2016;375:730-9. doi:10.1056/NEJMoa1600159

85 Gallay MN, Moser D, Rossi F, et al. Incisionless transcranial MR-guided focused ultrasound in essential tremor: cerebellothalamic tractotomy. Journal of Therapeutic Ultrasound 2016;4:5. doi:10.1186/s40349-016-0049-8

86 Schlesinger I, Eran A, Sinai A, et al. MRI Guided Focused Ultrasound Thalamotomy for Moderate-to-Severe Tremor in Parkinson's Disease. Parkinsons Dis 2015;2015:219149. doi:10.1155/2015/219149

87 Magara A, hler RB, Moser D, et al. First experience with MR-guided focused ultrasound in the treatment of Parkinson's disease. Journal of Therapeutic Ultrasound 2014;2:1-8. doi:10.1186/2050-5736-2-11

88 Kitagawa M, Murata J, Kikuchi S, et al. Deep brain stimulation of subthalamic area for severe proximal tremor. Neurology 2000;55:114-6.

89 Herzog J, Hamel W, Wenzelburger R, et al. Kinematic analysis of thalamic versus subthalamic neurostimulation in postural and intention tremor. Brain 2007;130:1608-25. doi:10.1093/brain/awm077

90 Groppa S, Herzog J, Falk D, et al. Physiological and anatomical decomposition of subthalamic neurostimulation effects in essential tremor. Brain 2014;137:109-21. doi:10.1093/brain/awt304

91 Plaha P, Patel NK, Gill SS. Stimulation of the subthalamic region for essential tremor. Journal of Neurosurgery 2004;101:48-54. doi:10.3171/jns.2004.101.1.0048

92 Barbe MT, Liebhart L, Runge M, et al. Deep brain stimulation in the nucleus ventralis intermedius in patients with essential tremor: habituation of tremor suppression. J Neurol 2011;258:434-9. doi:10.1007/s00415-0105773-3

93 Velasco FC. Other targets to treat Parkinson`s disease (posterior subthalamic targets and motor cortex). In: Lozano AM, Gildenberg PL, Tasker RR, eds. Textbook of sterotactic and functional Neurosurgery. J. Springer 2009. 1665-78.

94 Elble RJ, Pullman SL, Matsumoto JY, et al. Tremor amplitude is logarithmically related to 4- and 5-point tremor rating scales. Brain 2006;129:2660-6. doi:10.1093/brain/awl190 